\title{
Research Paper \\ Effect of a Sleep Hygiene Education Program Using Telephone Follow-up Method on the Sleep Quality of the Elderly
}

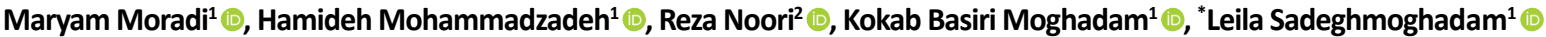

1. Social Development and Health Promotion Research Center, Gonabad University of Medical Sciences, Gonabad, Iran.

2. Department of Community Health and Management Nursing, Faculty of Nursing, Gonabad University of Medical Sciences, Gonabad, Iran.

\begin{tabular}{|c|c|}
\hline $\begin{array}{l}\text { Use your device to scan } \\
\text { and read the article online }\end{array}$ & Sadeghmoghadam I Effect of a Slee \\
\hline 口Esing & Education Program Using Telephone Follow-up Method on the Sleep Quality of the Elderly (Persian)]. Iranian Journal of Ageing. \\
\hline 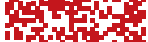 & 2021; 15(4):484-495. https://doi.org/10.32598/sija.15.4.2895.1 \\
\hline 口Fates & doi'https://doi.org/10.32598/sija.15.4.2895.1 \\
\hline
\end{tabular}

\section{(i) (\$)}

Received: 07 Apr 2020 Accepted: 06 Oct 2020 Available Online: 24 Feb 2021

Keywords: Aging, Sleep quality, Sleep health, Telephone follow-up

\section{A B S T R A C T}

Objectives Low quality sleep is one of the most common problems in old age. The present study aims to determine the effect of a sleep hygiene education program using Telephone Follow-Up (TFU) method on the sleep quality of the elderly.

Methods \& Materials This quasi-experimental study was conducted on 80 eligible elderly people aged 6074 years who were selected using a systematic random sampling method and were randomly assigned into intervention and control groups. The intervention group received a one-hour face-to-face education session on sleep hygiene. The TFU were carried out two times per week in the first month and once a week in the second month after intervention. The control group received routine care. The data collection tools were a demographic form and the Pittsburgh Sleep Quality Index (PSQI) questionnaire. The PSQI was completed for both groups immediately after the second month.

Results There was no statistically significant difference in the mean PSQI score before intervention in the intervention (8.02) and control (7.47) groups ( $\mathrm{P}=0.32)$, but after the intervention, the mean $\mathrm{PSQ}$ I score in the intervention group (5.42) was significantly reduced compared to the control group (7.67) $(P<0.001)$. Conclusion Sleep hygiene education program based on TFU improves the sleep quality of the elderly. It is recommended that health care providers use this method to improve the sleep quality of the elderly.

\section{Extended Abstract}

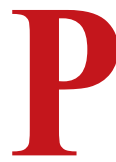

\section{Introduction}

oor quality sleep is one of the most common complaints of elderly people seeking medical attention. Due to the physiological changes of aging, the pharmacokinetics and pharmacodynamics of drugs also change; hence, the possibility of using chemical drugs becomes more limited. One of the non-pharmacological methods to improve sleep quality is sleep hygiene education program. Sleep hygiene means improving individual, behavioral and environmental factors that have negative effects on the sleep quality. Among the methods of care services, telephone follow-up (TFU) is a very useful method for assessing the needs for out-of-hours care of patients and reduces the number of frequent visits. It is a very useful, cheap and effective method in promoting self-care in patients that is less considered in Iran [6]. The aim of this study was to investigate the effect of sleep hygiene education program based on TFU on the sleep quality of the elderly covered by community health centers in Iran.

* Corresponding Author:

Leila Sadeghmoghadam, PhD.

Address: Social Development and Health Promotion Research Center, Gonabad University of Medical Sciences, Gonabad, Iran.

Tel: +98 (51) 57223028

E-mail: Is_moghadam@yahoo.com 


\section{Methods \& Materials}

This is a quasi-experimental study. Inclusion criteria were: consent to participate in the study, age 60-74 years, hearing and cognitive health, and the possibility of making telephone calls. Exclusion criteria were: Having depression, cancer, heart failure, severe lung disease, mental and physical disability, chronic pain, Alzheimer's according to the medical records, and hospitalization, death of relatives and any other similar unexpected events, and disconnection of telephone communication for 3 weeks during the intervention. The sample size was determined 40 older people for each group according to a similar study. Data collection tool was a two-part questionnaire, the first part surveys demographic information and the second part was the Pittsburgh Sleep Quality Index (PSQI).

The samples were randomly divided into control $(n=40)$ and intervention $(\mathrm{n}=40)$ groups. Then, for the intervention group, a one-hour face-to-face education session was held in the form of lectures, group discussions, questions and answers. In this session, educational content were presented in terms of the importance of sleep in the elderly, sleep changes in old age, and sleep hygiene program. After the education session, TFU was done in order to follow up and ensure behavior change in the elderly in the first month, twice a week, and in the second month every week (a total of 12 follow-ups). The telephone number was also provided to the elderly for counseling. Then, immediately after the second month, the elderly were invited to refer to community health centers to complete the questionnaire on certain days. In case of not coming, the researcher went to their house to distribute the questionnaires. For the control group, routine care was provided and an educational booklet was provided to them after the end of intervention.

\section{Results}

The mean age of participants was 68.18 years; $62.5 \%$ were female, $53.3 \%$ were housekeeper, $76.3 \%$ were married and most of them had primary education. The economic status of $72 \%$ of them was fair (sufficient to meet their living needs). Except for marital status, there was no statistically significant difference in demographic variables between the two groups. According to the independent statistical test results, there was no significant difference in the mean sleep quality scores between the two groups before the intervention $(\mathrm{P}>0.05)$. After the intervention, the difference between the two groups as well as the mean pre- and post-test difference was significant $(\mathrm{P}<0.001)$ (Table 1).

\section{Conclusion}

Sleep hygiene significantly improves the quality of sleep in the elderly. Therefore, due to the very high prevalence of insomnia and its complications in the elderly, we suggests sleep hygiene education as a non-pharmacological method for the management of sleep disorders in the elderly due to ease of use, having no complications, and improving the quality of life. This technique is more costeffective and time-consuming compared to sleep medications, and can improve the health of the elderly dwelling in the community. This method can be taught to health center staff, nurses as well as caregivers to be used for improving the sleep quality of older people. Further studies are recommended on investigating the effect of this educational method in the elderly living in nursing homes using longer follow-up periods.

\section{Ethical Considerations}

\section{Compliance with ethical guidelines}

This study was approved by Ethical Committee of Gonabad University of Medical Sciences (Code: IR.GMU.REC.1398.020). All ethical principles are considered in this article. The participants were informed about the purpose of the research and its implementation stages. They were also assured about the confidentiality of their information and were free to leave the study

Table 1. Comparing the mean sleep quality scores of subjects in both groups before and after intervention

\begin{tabular}{|c|c|c|c|}
\hline \multirow{2}{*}{ Sleep Quality } & \multicolumn{2}{|c|}{ Mean士SD } & \multirow{2}{*}{$\begin{array}{l}\text { Independent t-test Results } \\
\text { (P) }\end{array}$} \\
\hline & Intervention & Control & \\
\hline Pre-test & $62.3 \pm 02.8$ & $75.2 \pm 47.7$ & 44.0 \\
\hline Post-test & $41.2 \pm 42.5$ & $45.2 \pm 67.7$ & $<001.0$ \\
\hline Mean Dff. & $40.2 \pm 60.2$ & $23.2 \pm 20.0-$ & $<001.0$ \\
\hline
\end{tabular}


whenever they wished, and if desired, the research results would be available to them.

Funding

This article was extracted from a research project of the Social Development and Health Promotion Research Center of Gonabad University of Medical Sciences.

\section{Authors' contributions}

Conceptualization and supervision: Leila Sadeghmoghadam and Maryam Moradi; Methodology: Leila Sadeghmoghadam, Maryam Moradi; Investigation, writing - original draft, writing - review \& editing: All authors; Data collection: Reza Noori, Vahide Mohammadzade and Maryam Moradi; Data analysis: Kokab Basirimoghadam.

\section{Conflicts of interest}

The authors declared no conflict of interest.

\section{Acknowledgments}

The authors would like to thank the Social Development and Health Promotion Research Center and the Deputy for Research of Gonabad University of Medical Sciences and all participants for their cooperation. 


\title{
تأثير برنامه آموزش بهداشت خواب مبتنى بر ييگيرى تلفنى بر كيفيت خواب سالمندان
}

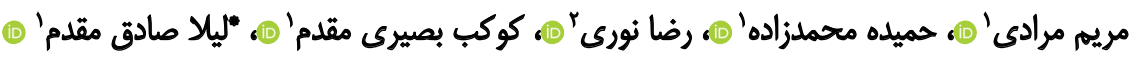

ا. مركز تحقيقات توسعه اجتماعى وارتقاى سلامت، دانشكاه علوميزشكى كناباد، كئاباده ايران.

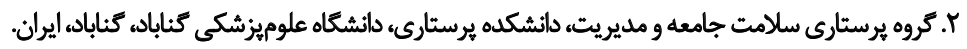

\begin{abstract}
حكبد

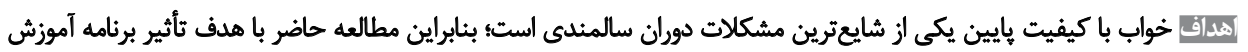

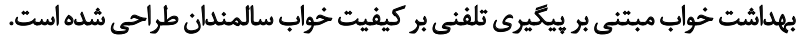

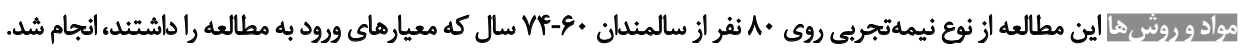

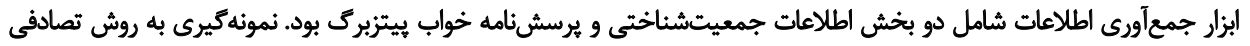

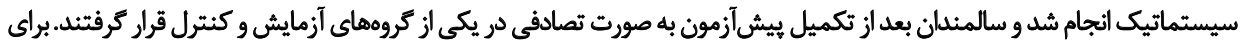

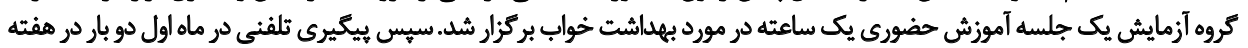

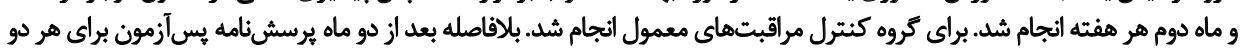

$$
\text { كروه تكميل شد. }
$$

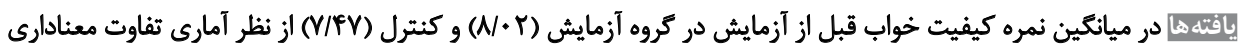

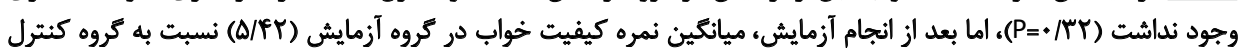

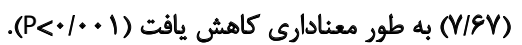

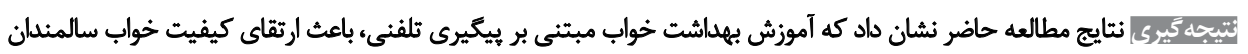

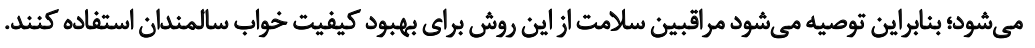

تاريخ دريافت: 19 فروردين

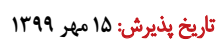
تاريخ انتشار: 9. اسفند

كليدوازوهها: - ن سالمند، كيفيث خواب،

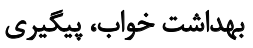
تلفنى

دارد و از جمله شكايات شايع و دلايل مراجعه افراد سالمند به

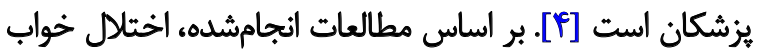

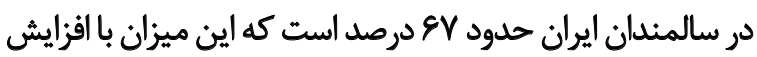
سن افزايش مىيابد [ه] خواب و استراحت نقش ترميمى و حفاظتى ايفا مي كند و در

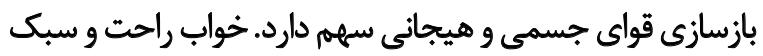

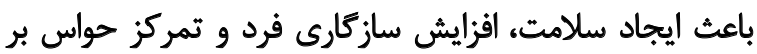

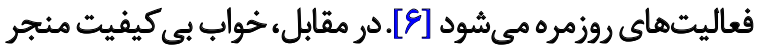

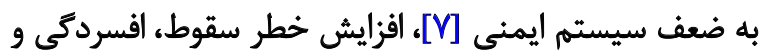

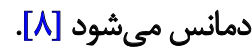

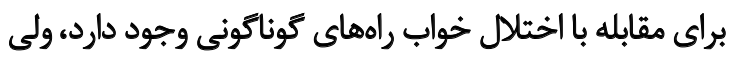

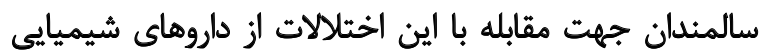

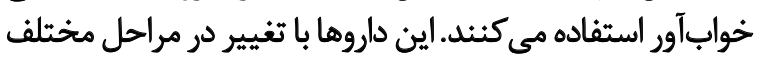

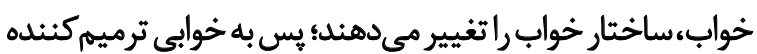

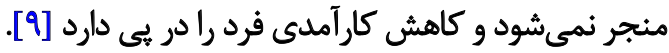

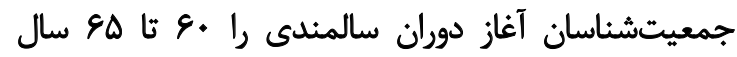

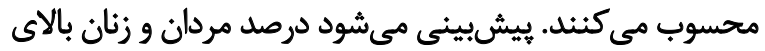

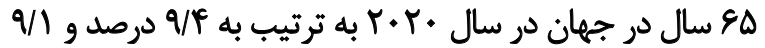
درصد افزايش يابد [1].] بر اساس آمار رسمى مركز آمار ايران، نتايج سرشمارى عمومى دمانى

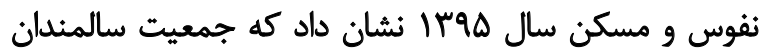

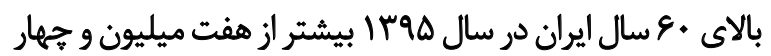

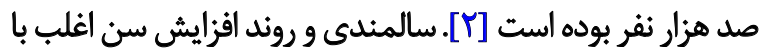

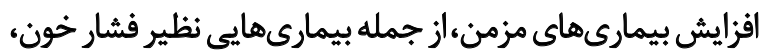

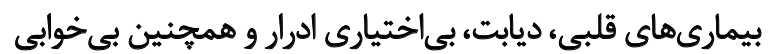
بروز مي كند [1]. طبق تحقيقات انجامشده، خواب با كيفيت بايين بعد از سردرد و اختلالات كوارشى، در رتبه سوم مشكلات سالمندان قرائ برار 
امروزه با ييشرفت روزافزون فناورى، استفاده از تلفن به عنوان

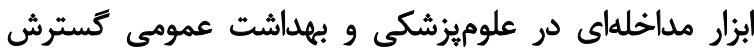

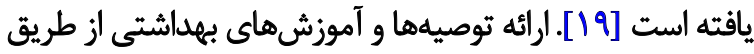

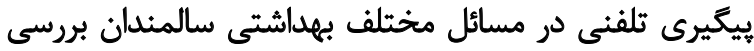

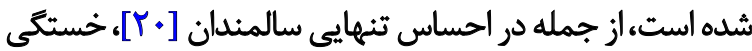

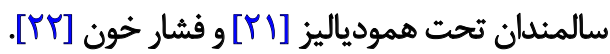

نتايج مطالعه مرورى سيستماتيك كه توسط شين و همكاران

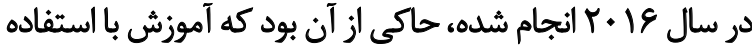

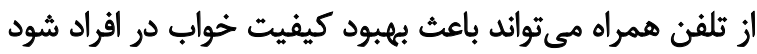

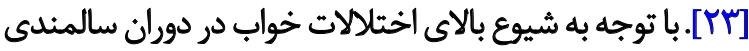

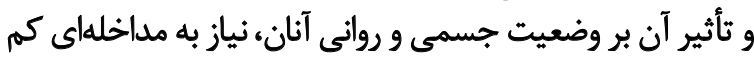
هزينه و بدون عارضه در اين زمينه احساس مى وشود. با توجه به اينكه بيشتر سالمندان داراى بيمارىهاى زمينداي

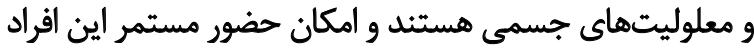

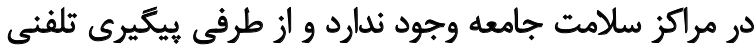

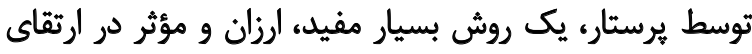

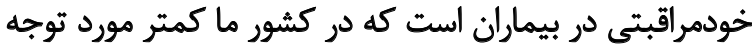

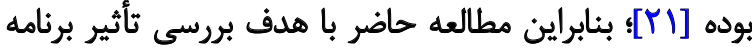

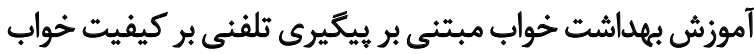
سالمثدان در مراكز سلامت جامعه شهرى شهر گتناباد طراحى

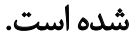
روش مطالعه

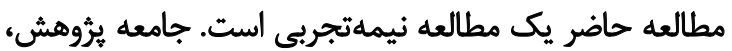

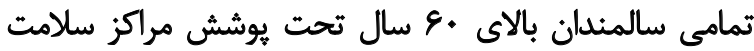

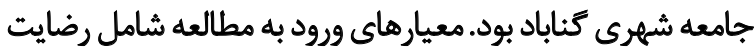

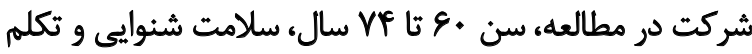
(از طريق بررسى يرونده بهداشتى و سؤال از سالمند و و مشاهدها)،

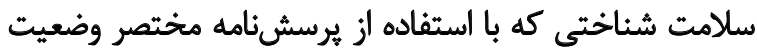

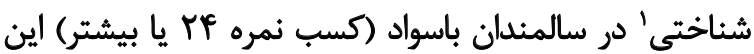

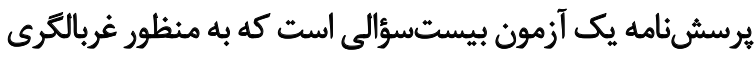

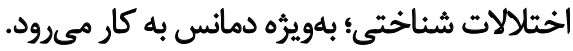
اين آزمون شدت و ميزان اختلالات شناختى راد يد يك زمان

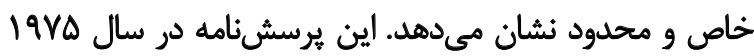

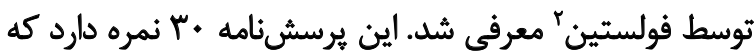

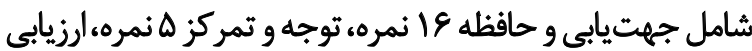

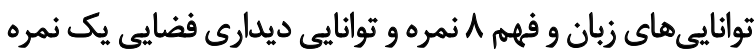

$$
\text { دارد. توانيب }
$$

نحوه امتيازدهى اين يرسشنامه به اين صورت است كه امتياز

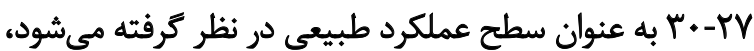

1. Mini Mental State Examination (MMSE)
با توجه به تغييرات فيزيولوريك سالمندى، فارماكوكينتيك

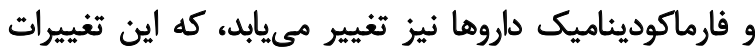

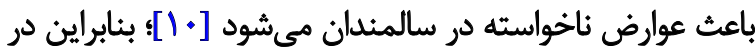

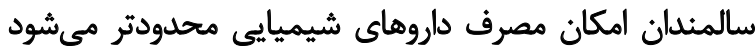

با اينكه اثربخشى روشهاي غيردارويى كندتر از اثربخشى

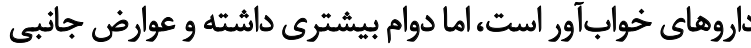

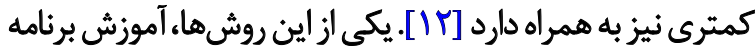

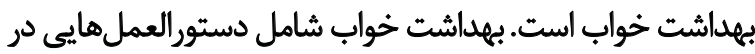
مورد، تعديل فاكتورهاى محيطى (روشنايى، دما و و سروصداب) و و

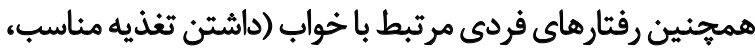

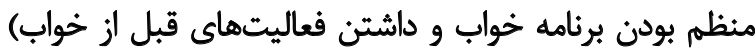

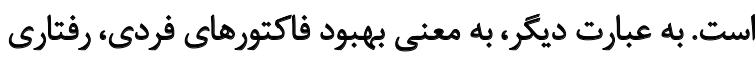

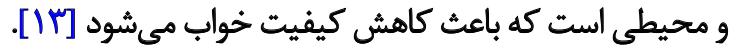
در خصوص تأثير آموزش برنامه بهداشت خواب روى كيفيت

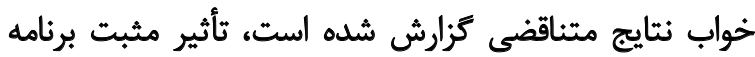

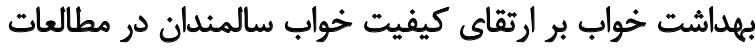
طاهرى و همكاران [If

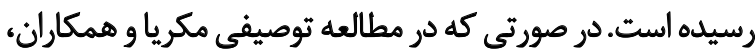

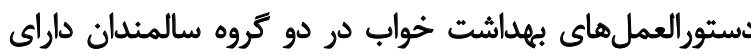

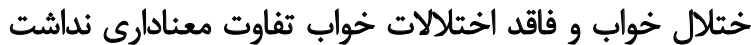

اكر حه بر اساس بثروهشهاي متعدد، به تأثير بهداشت خواب

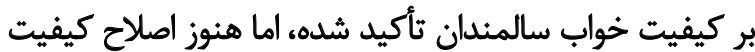

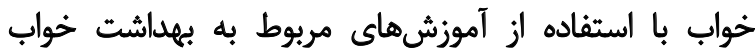

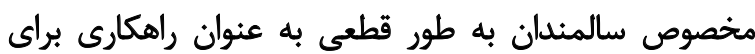

مواجهه با اختلالات خواب اين قشر از جامعه به كار نمىرود. شايد به دليل اينكه بهداشت خواب جزئى از درمان رفتارى

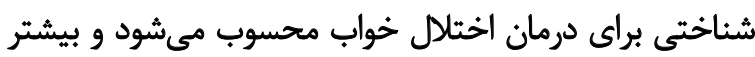

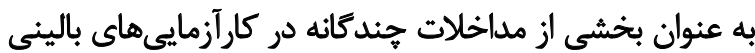

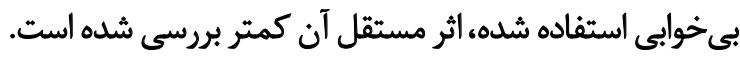
با توجه به مطالب ياد شده و مزيتهايي كه استفاده از آموزش

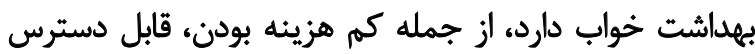

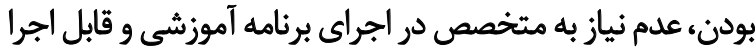

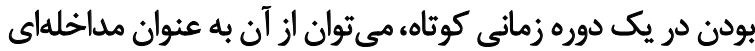

مناسب جهت ارتقاي كيفيت خواب سالمندان استفاده كردان يُيخيرى مستمر به عنوان بخش اساسى از سرويس هاي مراقبتى

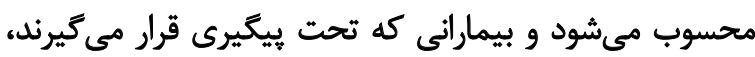

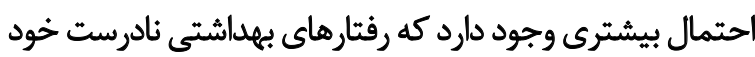

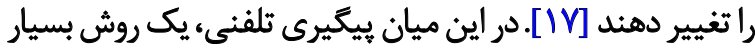

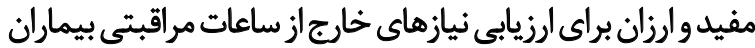

است و باعث كاهش تعداد ويزيتهاى مكرر مي شئود [1/1)]. 


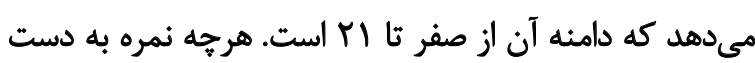

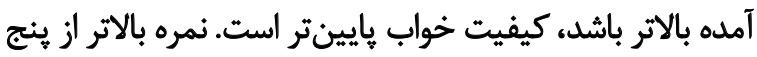

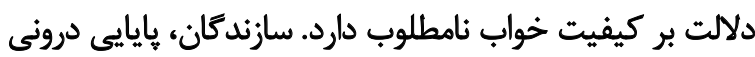

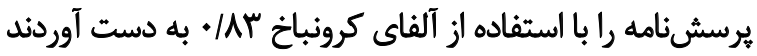

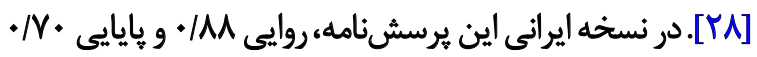

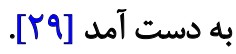

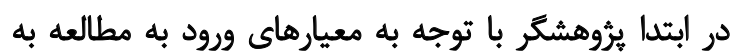

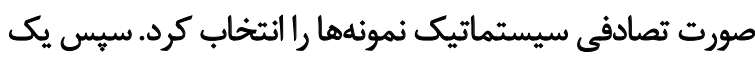

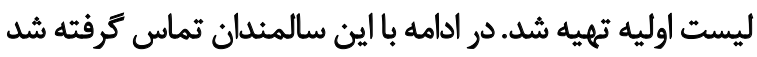

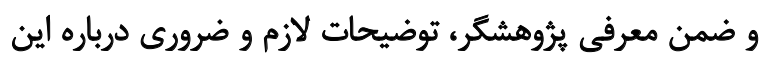
ئروهش به آنان ارائه شد.

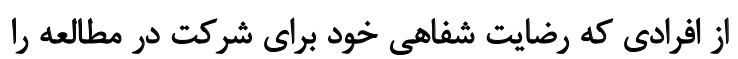

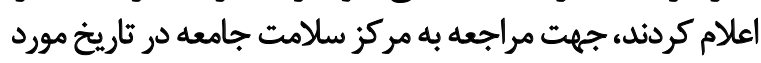

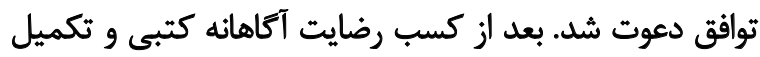

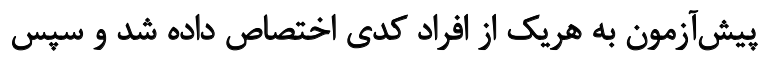

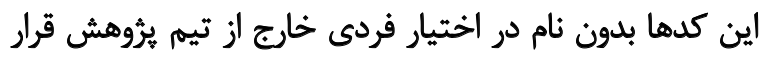

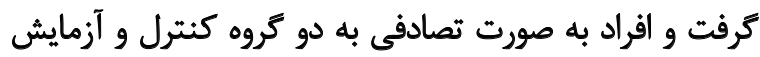

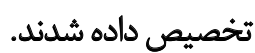

سيس افراد كروه آزمايش به دو كروه بيستنفره تقسيمبندي

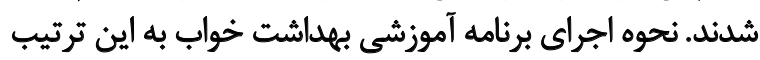

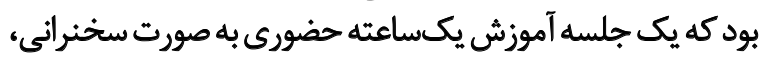

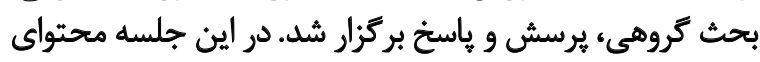

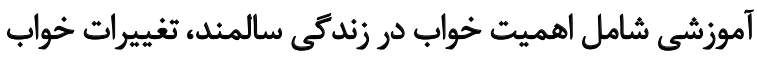

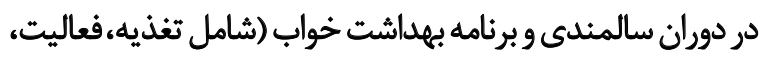

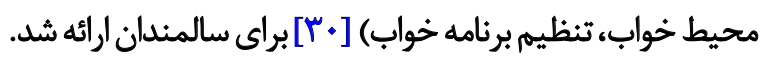

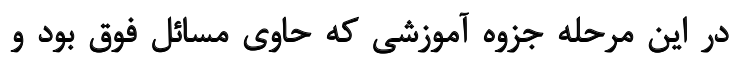

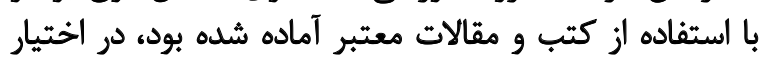

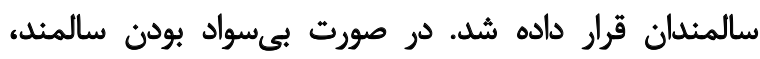

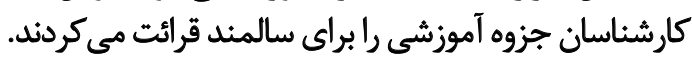

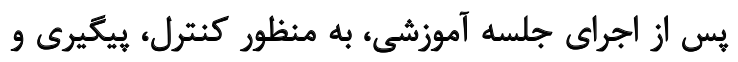

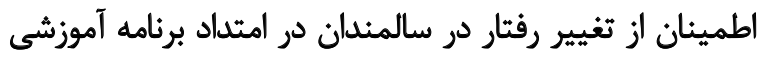

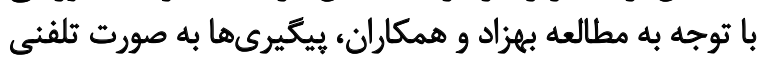

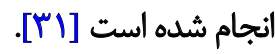

مشاورههاى مراقبت بيكير به صورت تلفنى در ماه اول بيكيرى،

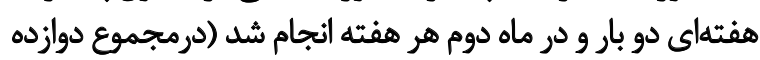

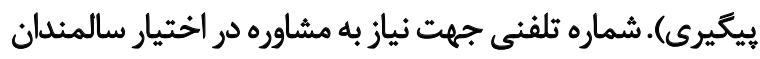

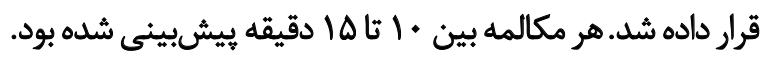

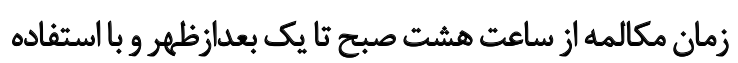

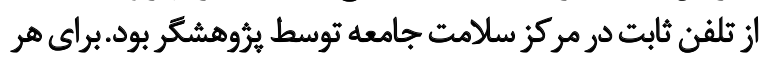

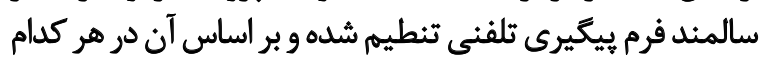

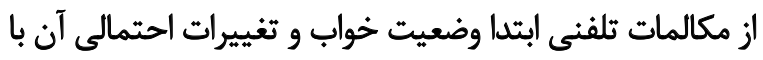

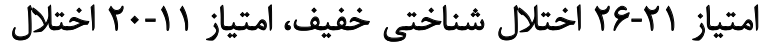

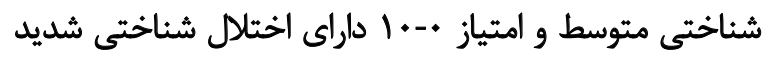

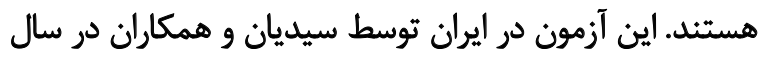

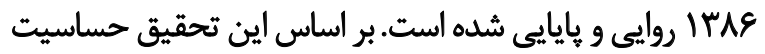

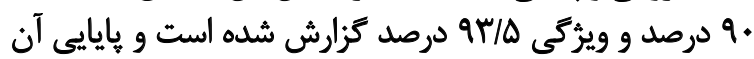

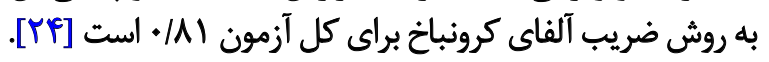

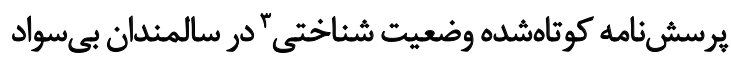

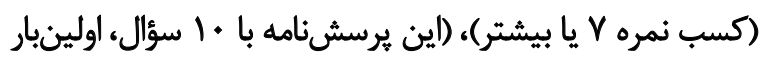

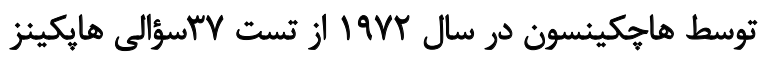

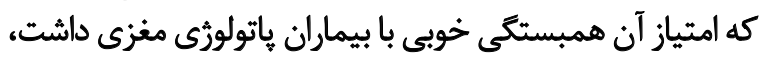

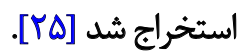

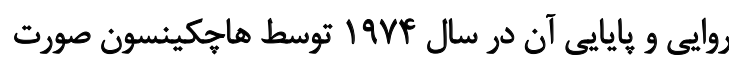

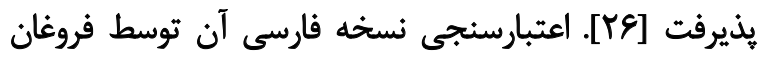

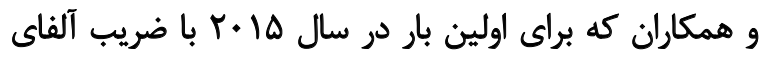

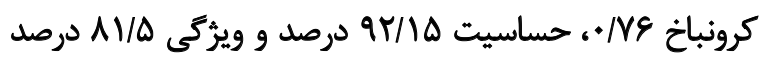

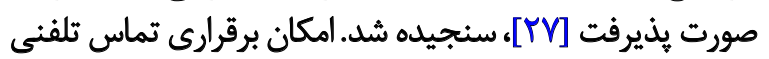

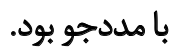

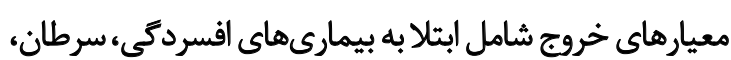

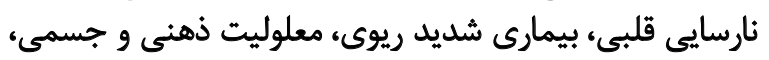

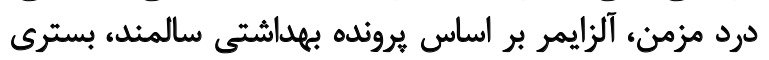

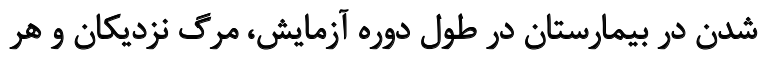

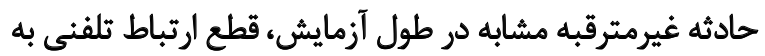
مدت سه هفته هنخام انجام آزمايش انتخاب شائ شدند.

ور اين مطالعه، حجم نمونه با استفاده از نرمافزار

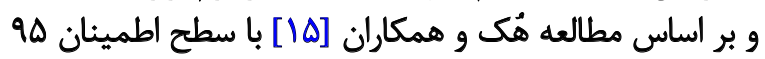

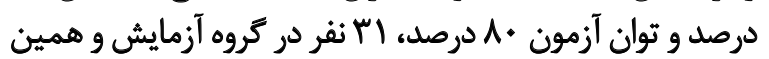

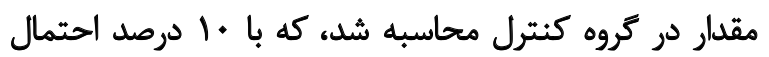

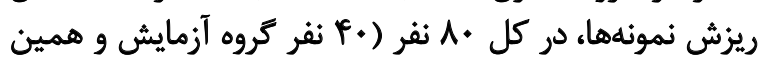

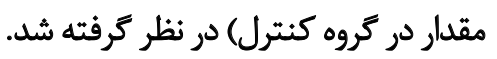

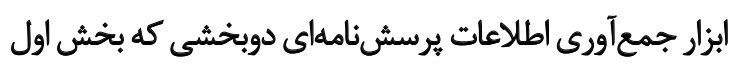

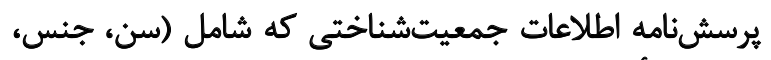

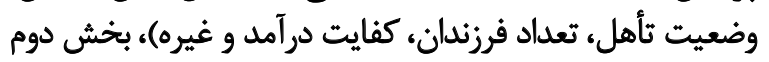

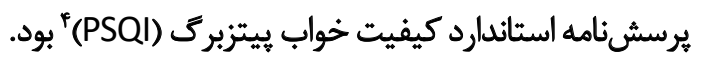

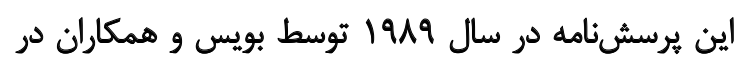

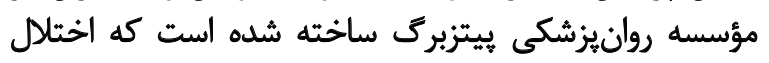

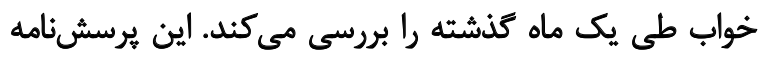

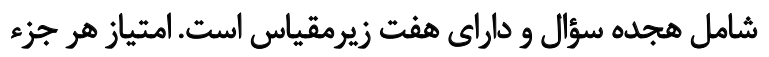
بين صفر تا سه است. مجموع ميانگين نمرات اين هفت جزء، نمره كل ابزار راتشكيل

3. Abbreviated Mental Test (AMT) 4. Pittsburgh Sleep Quality Index 
جدول ا. برئامه آموزشى بهداشت خواب

\section{برنامه آموزشى بهداشت خواب}

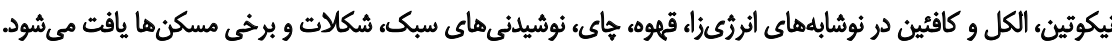

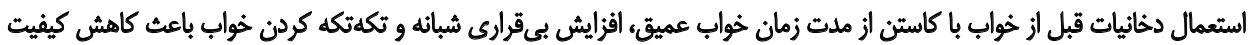
خواب فرد مي أشود. تعذيه

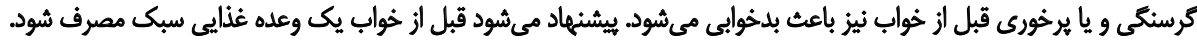

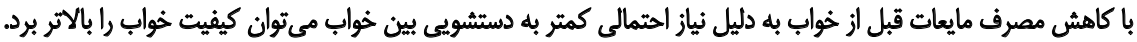

تمرينها و ورزش هاى فكرى و جسمى در زمانهاى ثزديك به زئ زمان خواب انجام داده نشود.

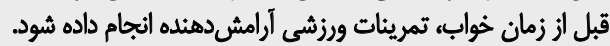

محيط خواب خنكه، آرام و بسيار تاريك باشد.

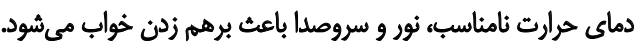

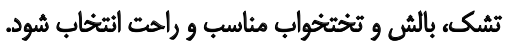

محيط خواب

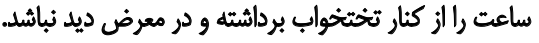

در نظر كرفتن زمان خاص براى خوايبين و رعايت آن

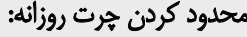

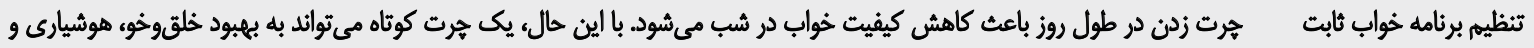

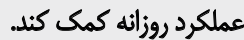

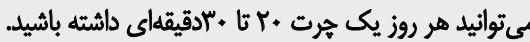

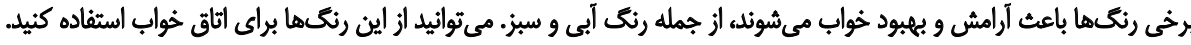

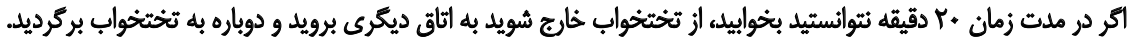

طراحى مناسب اثتاق خواب

L

$$
\text { تى مستقل و آناليز واريانس يكسرفه انجام شد. }
$$

ياقتهلها

در اين مطالعه، اطلاعات مربوط به هشتاد سالمند (شامل

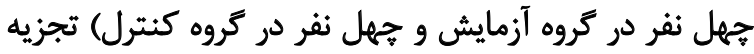

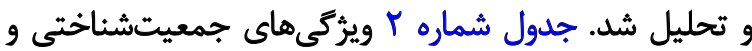

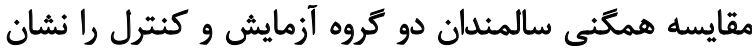

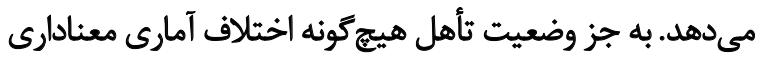

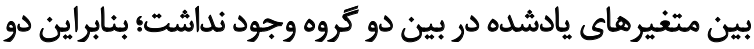

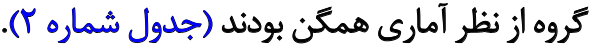
بر اساس آزمون آمارى تى مستقل، بين ميانكين نمره كيفيت

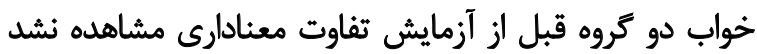

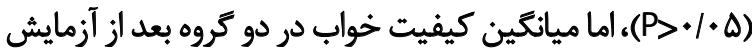

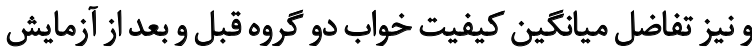

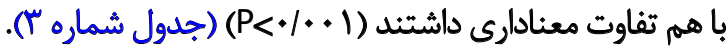

هدف از يُوهش حاضر تأثير برنامه آموزش بهداشت خواب تئي

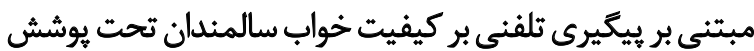

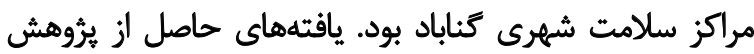

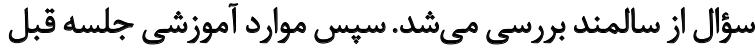

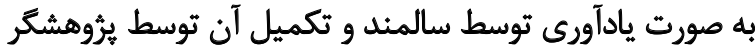

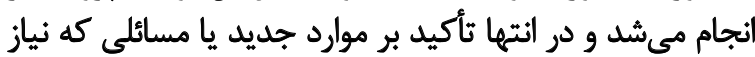

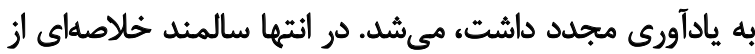

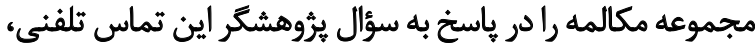

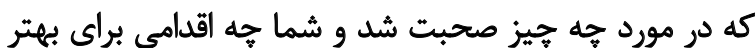

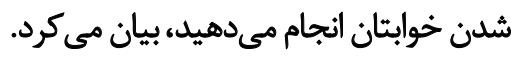
در هر جلسه مكالمه، اين مرحله تكرار مى شيد و در فرم بيعيرى

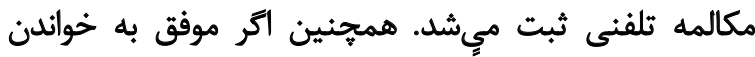

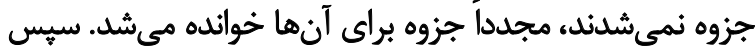

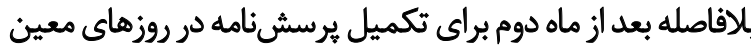

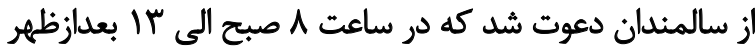

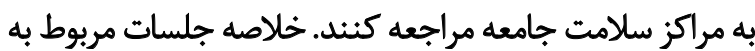

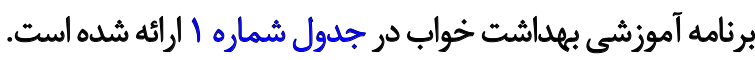

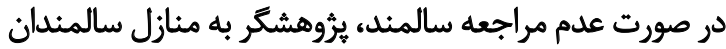

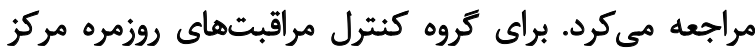

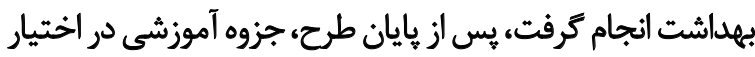
سالمندان كروه كنترل قرار داده شد.

در اين بُروهش براى تجزيه و تحليل دادهها با استفاده از آمار

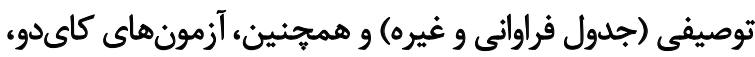


جدول r. مقايسه ويرّكى هاى جمعيتشناختى سالمندان در كروه آزمايش وكنترل

\begin{tabular}{|c|c|c|c|c|}
\hline \multirow{2}{*}{$\mathbf{P}$} & كثترل & أزمايش & \multirow{2}{*}{ متغير } & \multirow{2}{*}{ كروه } \\
\hline & ن ثلأحراف معيار & فراوانى & & \\
\hline \multirow{2}{*}{.$/ / V^{*}$} & $r \cdot(F Y / P)$ & If $(r \Delta)$ & مرد & \multirow[b]{2}{*}{ جنسيت } \\
\hline & $r+(\Delta S / N)$ & $r \varepsilon(\& \Delta)$ & زن & \\
\hline \multirow{3}{*}{.$/ \mathrm{Fr}$} & $r \cdot(\Delta *)$ & $\operatorname{rf}\left(\varepsilon_{0}\right)$ & خانهدار & \multirow{3}{*}{ اشتغال } \\
\hline & $9(Y r / Y)$ & $\Delta(I K / \Delta)$ & آزاد & \\
\hline & $\|$ (TV/A) & $\|(T V / \Delta)$ & بازتشسته & \\
\hline \multirow{2}{*}{$.1 \cdot 1^{*}$} & $r q(N / \Delta)$ & $r \cdot(v \Delta)$ & مثتاهل & \multirow{2}{*}{ تأهل } \\
\hline & $\|(W / Q)$ & $1 \cdot(T \Delta)$ & بلون همسر & \\
\hline \multirow{2}{*}{. ra* } & $\pi(A \Delta)$ & $M V(q Y / Q)$ & با خائواده & \multirow{2}{*}{ همراه زندكى سالمند } \\
\hline & $8(10)$ & $r(V / \Delta)$ & به تنهايي & \\
\hline \multirow{3}{*}{.$/ F \Delta^{*}$} & $\Delta(I T / \Delta)$ & $1 .(T A)$ & كمتر از تأمين نياز & \multirow{3}{*}{ وضعيت اقتصادى } \\
\hline & & & & \\
\hline & $\Gamma \Delta(A V / \Delta)$ & $r \cdot(V Q)$ & در حلد تأمين ثياز & \\
\hline $.1 \cdot v^{* *}$ & \multicolumn{2}{|c|}{ EVRTY $\pm \Delta / \Delta}$. & $8 \Delta / \cdot \Delta \pm p^{e} / \mathrm{r}$ & سن \\
\hline $.18 q^{* * *}$ & \multicolumn{2}{|c|}{$\varphi / A+ \pm r / \cdot r$} & $\Delta / T+ \pm F / P \Delta$ & تحصيلات \\
\hline
\end{tabular}

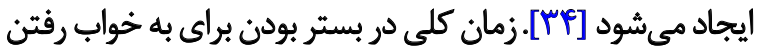

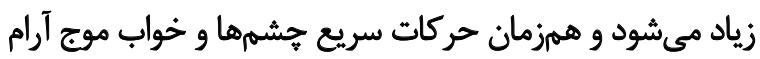

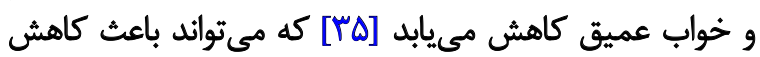
كيفيت خواب در سالمندان شود.

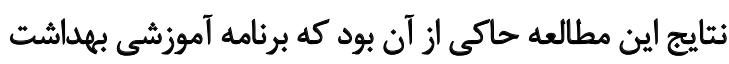

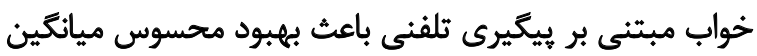

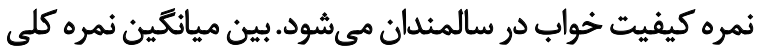

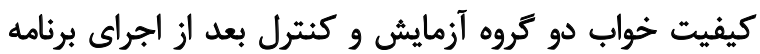

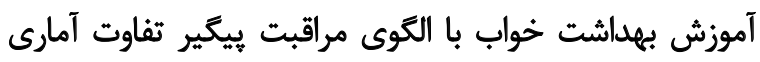

حاكى از إيين بودن ميانكين نمره كلى كيفيت خواب سالمندان در دو كروه آزمايش و كنترل قبل ازئ انجام آزمايش بوديف بودي

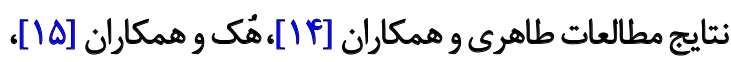

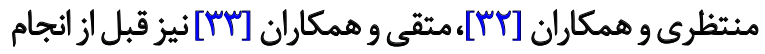

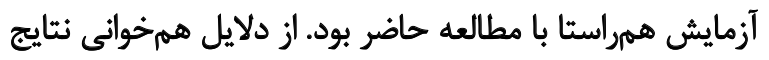

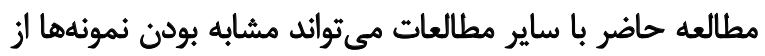
نظر كروه سنى باشد.

بر اساس مطالعات انجامشده با بالا رفتن سن، تغييرات عمده

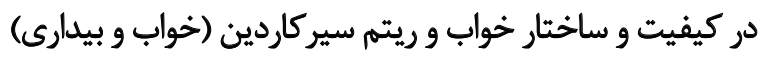

جدول ب. ميانكين نمره و تفاضل ميانكين نمره كيفيت خواب در دو كروه مداخله و كنترل قبل و بعد از اجراى برنامه آموزشى بهداشت خواب مبتنى بر بيكيرى ثلفنى در سالمندان

\begin{tabular}{|c|c|c|c|c|}
\hline$P$ & \multicolumn{2}{|c|}{ انحراف معيار 土ميانكين } & \multirow{2}{*}{\multicolumn{2}{|c|}{ 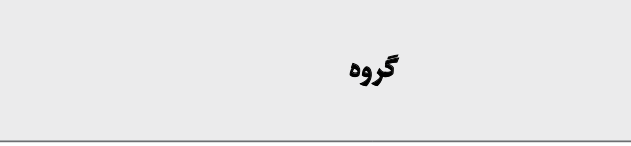 }} \\
\hline نتايج آزمون تى مستقل & كروه كثترل & كروه مداخله & & \\
\hline.$/ \mu$ & $V / P V \pm T / V \Delta$ & $N T \cdot \pm r / g r$ & ق ق ق ازل مداخله & \\
\hline$>\cdot 1 . .1$ & $V / F V \pm r / P Q$ & $\Delta / \varphi r \pm T / \varphi)$ & ميس از هداخله & كيقيت خواب \\
\hline$>\cdot 1 \cdot .1$ & $-\cdot \pi \cdot \pm T / \pi$ & $r / \varepsilon \cdot \pm T / \varphi \cdot$ & تفاضل ميانكين قبل و يس از مداخله & \\
\hline
\end{tabular}

L 
نتايج مطالعات مختلف حاكى از آن است كه آموزش و ييكيرى

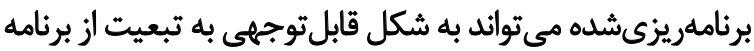

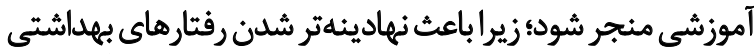

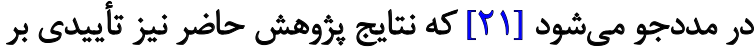
اين مطالعات است.

در بيشتر مطالعاتى كه يرُوهشكَر جستوجو كرد، نتايج

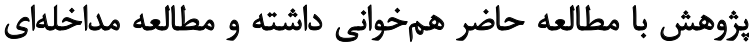

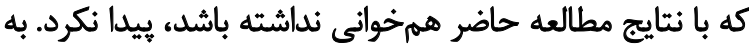

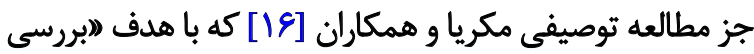

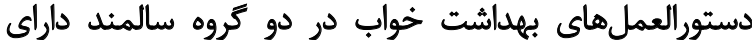
اختلال خواب و فاقد اختلال خوابه) انجام شد.

نتايج حاكى از آن بود كه بهداشت خواب در دو گروه سالمندان

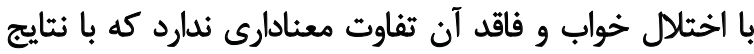

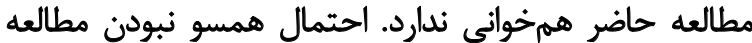

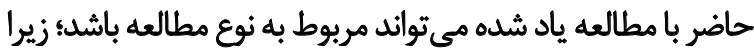

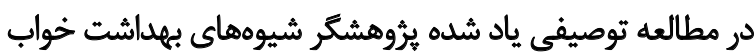

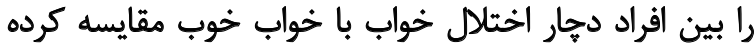

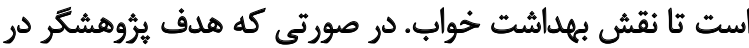

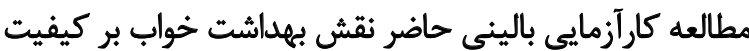

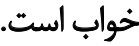

\section{نتيجلمَيرى نههايى}

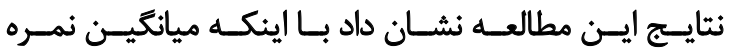

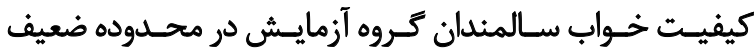

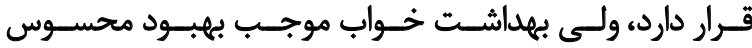

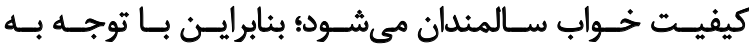

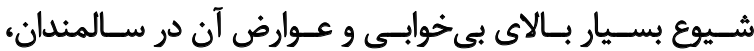

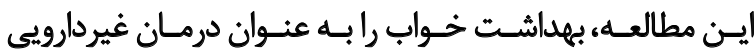

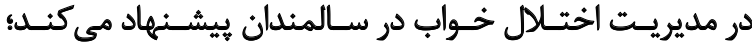

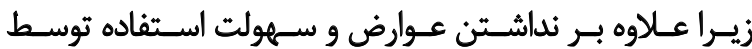

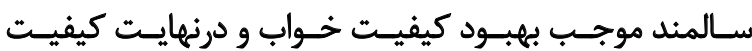

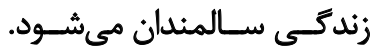

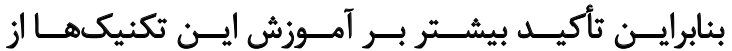

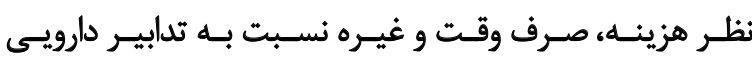

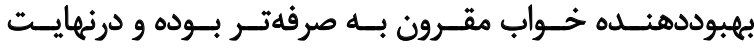

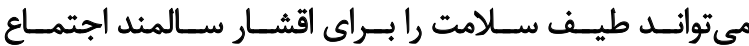

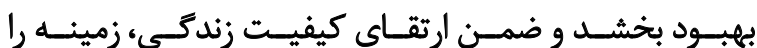

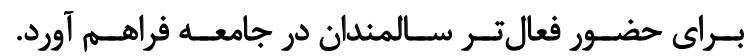

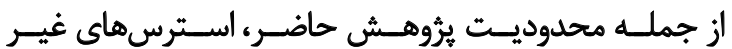

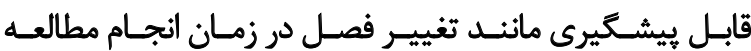

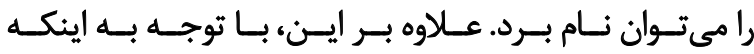

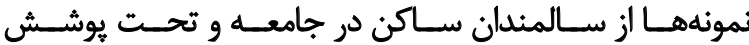

معنادارى مشاهده شد.

مطالعات متعددى همراستا با نتايج بهدستآمده از يرؤهش

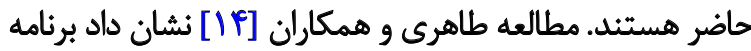

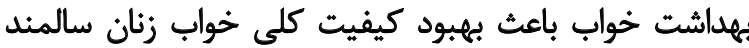

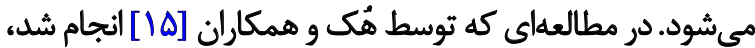
نتايج نشان داد كه برنامه بهداشت خواب توداب باعث بهبود

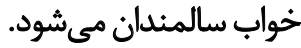

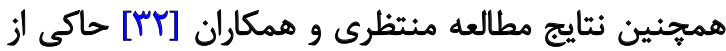

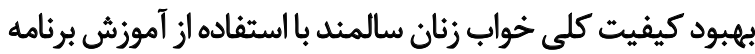

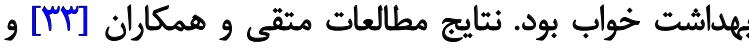

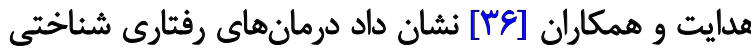

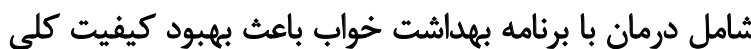

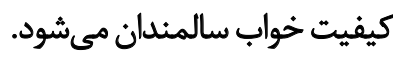

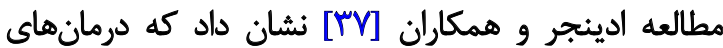

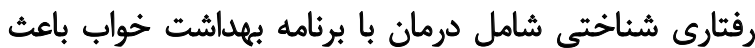

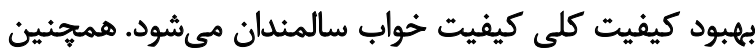

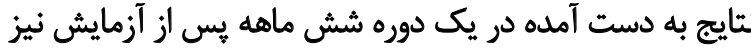

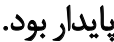

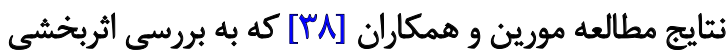

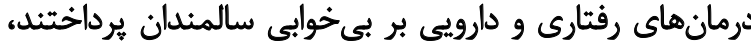

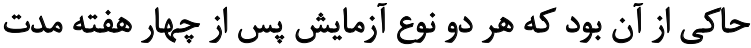

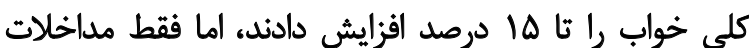

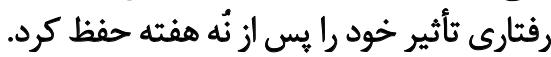

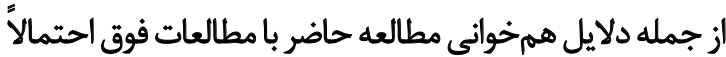

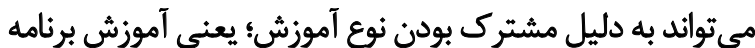

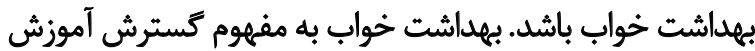

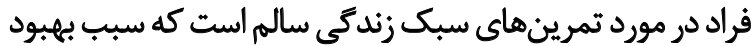

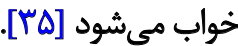
بر اساس تحقيقات انجامشده برنامه بهداشت خواب، علاوه بر بر برائ برائ

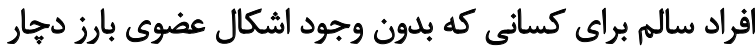

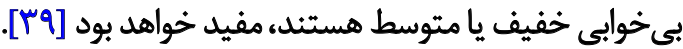
نتايج مطالعه بهزاد و همكاران، حاكى از آن بود كه برنامئه

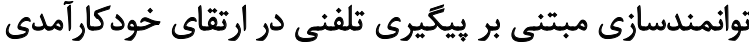

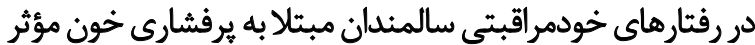

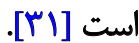

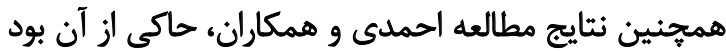

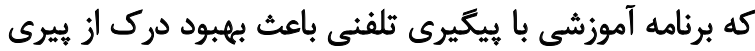

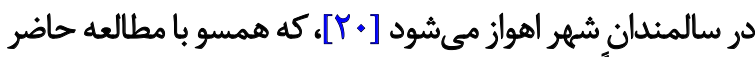

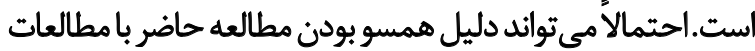
فوق مشابه بودن نوع يُيكيرى باشد. 


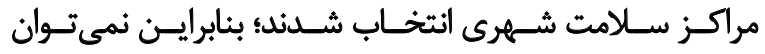

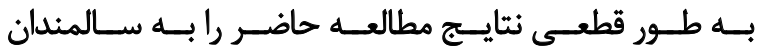
روسـتايى و سـاكن خاتسه سـالمندان تعميـم ماد.

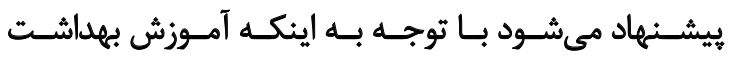

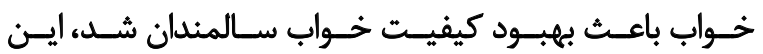

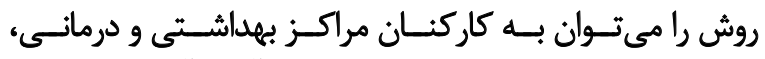

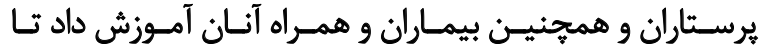

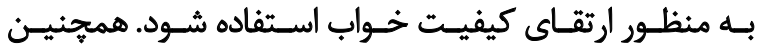

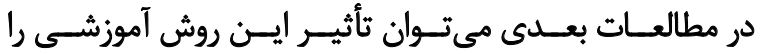

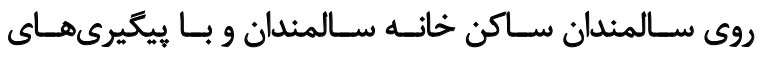
طولانىتــر بررسى كـرد.

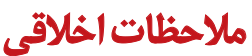

يبيروى أز اصول اخلاق يُونش

تمامى اصول اخلافى اين يُروهش مورد تاييد كميته اخلا

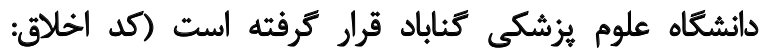

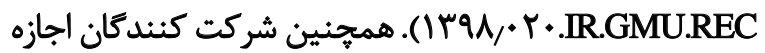

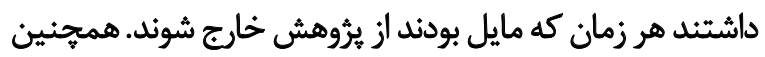

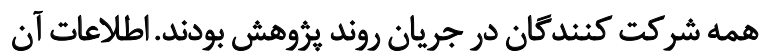
ها محرمانه نتكه داشته شد شند.

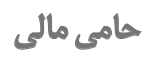

اين مقاله حاصل استخراج از طرح تحقيقاتى است كه در مركز

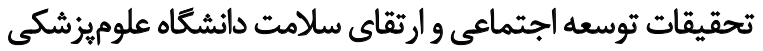
كناباد تصويب شده است.

\section{مشاركت ثويسندكًان}

مفهومسازى و ايده اصلى: ليلا صادق مقدم، مريم مرادى؛ روش

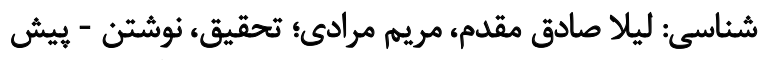

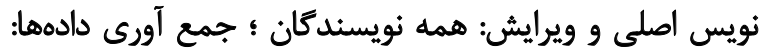
رضانورى، وحيد محمدزاده، مريم مرادي؛ تجزيه و وتحليل واديل دادهها:

$$
\text { كوكاب بصيرىمقدم. }
$$

$$
\text { تعارض مثاقع }
$$

بنابر اظهار نويسندكان اين مقاله هيجَّونه تعارض منافعى

$$
\text { تشكر وقدرداثى }
$$

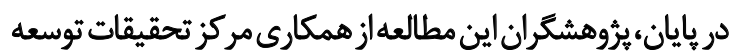

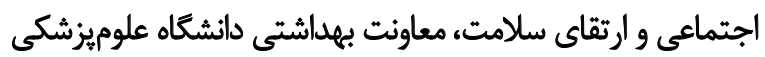
كناباد و سالمندان كمال سياس كزارى را به عمل مى آورند. 


\section{References}

[1] WHO. World report on ageing and health [Internet]. 2015 [Updated 2015 September 30]. Available from: https://www. who.int/ageing/events/world-report-2015-launch/en/

[2] Iran Statistical Center. Results of the Population and Housing Census of 2016 [Internet]. 2016 [Updated 2021]. Available from: https:/ / www.amar.org.ir/english/

[3] Shirazi Khah M, Mousavi M, Sahaf R. [Study of health and social indicators of elderly women in Iran (Persian)]. Iranian Journal of Ageing. 2012; 6(S1):66-78. http://salmandj. uswr.ac.ir/article-1-481-en.htm

[4] Bahrami M, Dehdashti AR, Karami M. [A survey on sleep quality in elderly people living in a nursing home in Damghan city in 2017: A short report (Persian)]. Journal of Rafsanjan University of Medical Sciences. 2017; 16(6):58190. http://eprints.rums.ac.ir/5501/

[5] Mirzaei M, Gholamrezaei E, Bidaki R, Fallahzadeh H, Ravaei J. [Quality of sleep and methods of management of sleep disorders in elderly of Yazd city in 2016 (Persian)]. Journal of Shahid Sadoughi University of Medical sciences. 2017; 25(6):467-75. http://jssu.ssu.ac.ir/article-1-4233-en. html

[6] Thase ME. Correlates and consequences of chronic insomnia. General Hospital Psychiatry. 2005; 27(2):100-12. [DOI:10.1016/j.genhosppsych.2004.09.006] [PMID]

[7] Safa A, Adib-Hajbaghery M, Fazel-Darbandi A. [The relationship between sleep quality and quality of life in older adults (Persian)]. Journal of Nursing Education. 2015; 3(3):53-62. http://ijpn.ir/article-1-617-en.html

[8] Papi S, Karimi Z, Ghaed Amini Harooni G, Nazarpour A, Shahry P. [Determining the prevalence of sleep disorder and its predictors among elderly residents of nursing homes of Ahvaz city in 2017 (Persian)]. Salmand: Iranian Journal of Ageing. 2019; 13(5):576-87. [DOI:10.32598/ SIJA.13.Special-Issue.576]

[9] Ashrafi H, Ansarin K, Hasanzadeh M, Jouyban A. [Review on sleep disorders and their management (Persian)]. Medical Journal of Tabriz University of Medical Sciences and Health Services. 2018; 40(2):95-105. https:/ / mj.tbzmed. ac.ir/Article/22727

[10] Hosseini L, Hajibabaee F, Navab E. [Reviewing polypharmacy in elderly (Persian)]. Iranian Journal of Systematic Review in Medical Sciences. 2017; 1(1):15-24. http://ijsr.ir/ article-1-28-en.html

[11] Rowe TA, Juthani-Mehta M. Urinary tract infection in older adults. Aging Health. 2013; 9(5):519-28. [DOI:10.2217/ ahe.13.38] [PMID] [PMCID]

[12] Bagheri-Nesami M, Meskini L, Rostami-ghadi M. [The effect of aromatherapy with essential oils of lavender on sleep quality in the elderly (Persian)]. Journal of Gerontology. 2019; 4(2):63-73. [DOI:10.29252/joge.4.2.63]

[13] Van der Heijden KB, Smits MG, Gunning WB. Sleep hygiene and actigraphically evaluated sleep characteristics in children with ADHD and chronic sleep onset insomnia. Journal of Sleep Research. 2006; 15(1):55-62. [DOI:10.1111/ j.1365-2869.2006.00491.x] [PMID]
[14] Taheri Tanjani P, Khodabakhshi H, Etemad K, Mohammadi $\mathrm{M}$. [Effect of sleep hygiene education on sleep quality and general health of elderly women with sleep disorders living in birjand city, Iran, in 2016 (Persian)]. Iranian Journal of Ageing. 2019; 14(2):248-59. [DOI: 10.32598/sija.13.10.290]

[15] Hoch CC, Reynolds III CF, Buysse DJ, Monk TH, Nowell P, Begley AE, et al. Protecting sleep quality in later life: A pilot study of bed restriction and sleep hygiene. The Journals of Gerontology Series B: Psychological Sciences and Social Sciences. 2001; 56(1):P52-9. [DOI:10.1093/geronb/56.1.P52] [PMID]

[16] McCrae CS, Rowe MA, Dautovich ND, Lichstein KL, Durrence $\mathrm{HH}$, Riedel BW, et al. Sleep hygiene practices in two community dwelling samples of older adults. Sleep. 2006; 29(12):1551-60 [DOI:10.1093/sleep/29.12.1551] [PMID]

[17] Morin CM, Benca R. Chronic insomnia. The Lancet. 2012; 379(9821):1129-41. [DOI:10.1016/S0140-6736(11)60750-2]

[18] Wong KW, Wong FK, Chan MF. Effects of nurse-initiated telephone follow-up on self-efficacy among patients with chronic obstructive pulmonary disease. Journal of Advanced Nursing. 2005; 49(2):210-22. [DOI:10.1111/j.1365-2648.2004.03280.x] [PMID]

[19] McSwain SD, Bernard J, Burke Jr BL, Cole SL, Dharmar M, Hall-Barrow J, et al. American Telemedicine Association operating procedures for pediatric telehealth. Telemedicine and eHealth. 2017; 23(9):699-706. [DOI:10.1089/tmj.2017.0176] [PMID]

[20] Sadeghmoghadam L, Ahmadi Babadi S, Delshad Noghabi A Nazari S, Farhadi A. Effect of telenursing on aging perception of Iranian older adults. Educational Gerontology. 2019; 45(7):476-82 [DOI:10.1080/03601277.2019.1657263]

[21] Fallahpour S, Nasiri M, Fotokian Z, Jannat Alipour Z, Hajiabad $M$. [The effect of telephone follow up (tele-nursing) on fatigue in the elderly undergoing hemodialysis (Persian)]. Journal of Critical Care Nursing. 2018; 11(4):1-10. http://jccnursing.com/article1-437-fa.pdf

[22] Behzad Y, Bastani F, Haghani H. [Effect of empowerment program with the telephone follow-up (tele-nursing) on self-efficacy in self-care behaviors in hypertensive older adults (Persian)]. The Journal of Urmia Nursing and Midwifery Faculty. 2016 13(11):1004-15. http:/ / unmf.umsu.ac.ir/article-1-2422-en.html

[23] Shin JC, Kim J, Grigsby-Toussaint D. Mobile phone interventions for sleep disorders and sleep quality: systematic review. JMIR mHealth and uHealth. 2017; 5(9):e131. [DOI:10.2196/ mhealth.7244] [PMID] [PMCID]

[24] Seyedian M, Falah M, Nourouzian M, Nejat S, Delavar A Ghasemzadeh HA. [Validity of the Farsi version of mini-mental state examination (Persian)]. Journal of Medical Council of Iran 2008; 25(4):408-14. https://www.sid.ir/en/journal/ViewPaper. aspx?id=133666

[25] Hodkinson H. Evaluation of a mental test score for assessment of mental impairment in the elderly. Age and Ageing. 1972; 1(4):233-8. [DOI:10.1093/ageing/1.4.233] [PMID]

[26] Burns A, Lawlor B, Craig S. Rating scales in old age psychiatry. The British Journal of Psychiatry. 2002; 180(2):161-7. [DOI:10.1192/bjp.180.2.161]

[27] Foroughan M, Hossieni MA, Rashedi V, Rezaei M, Lotfi G. [Comparison of cognitive function in the monolingual and bilingual elderly (Persian)]. Journal of Kermanshah University of Medical Sciences. 2015; 18(10):607-9. [DOI:10.22110/jkums. v18i10.2299] 
[28] Buysse DJ, Reynolds CF, Monk TH, Berman SR, Kupfer DJ. The Pittsburgh Sleep Quality Index: a new instrument for psychiatric practice and research. Psychiatry Research. 1989; 28(2):193-213. [DOI:10.1016/0165-1781(89)90047-4]

[29] Hossein-Abadi R, Tabrizi K, Poor-Esmaiel Z, Karimloo M, Maddah S. [The effects of pressure massage on geriatrics sleep quality (Persian)]. Journal of Rehabilitation. 2008; 9(2):8-14. http://rehabilitationj.uswr.ac.ir/article-1-247-fa.html

[30] Trauer JM, Qian MY, Doyle JS, Rajaratnam SM, Cunnington D. Cognitive behavioral therapy for chronic insomnia: A systematic review and meta-analysis. Annals of Internal Medicine. 2015; 163(3):191-204. [DOI:10.7326/M14-2841] [PMID]

[31] Behzad Y, Hagani H, Bastani F. [Effect of empowerment program with the telephone follow-up (tele-nursing) on self-efficacy in self-care behaviors in hypertensive older adults (Persian)]. The Journal of Urmia Nursing and Midwifery Faculty. 2016; 13(11):1004-15. http:/ / unmf.umsu.ac.ir/article-1-2422-en.html

[32] Montazeri lemrasky M, Shamsalinia A, Hajiahmadi M, Nasiri M. [The Effectiveness of Education on Factors Affecting Elderly Women's Sleep (Persian)]. Journal of Caspian Health and Aging. 2018; 3(1):59-68. [DOI: 10.22088/cjhaa.3.1.59]

[33] Mottaghi R, Kamkar A, Maredpoor A. [Effectiveness of cognitive behavior therapy on the quality of sleep in elderly people with insomnia disorder (Persian)]. Salmand: Iranian Journal of Ageing. 2016; 11(2):234-43. [DOI:10.21859/sija-1102234]

[34] Akbari Kamrani AA, Shams A, Abdoli B, Shamsipour Dehkordi $P$, Mohajeri R. The effect of low and moderate intensity aerobic exercises on sleep quality in older adults (Persian)]. Salmand: Iranian Journal of Ageing. 2015; 10(1):72-81. http:/ / salmandj.uswr. ac.ir/article-1-712-en.html

[35] Gleason K, McCall WV. Current concepts in the diagnosis and treatment of sleep disorders in the elderly. Current Psychiatry Reports. 2015; 17(6):45. [DOI:10.1007/s11920-015-0583-z] [PMID]

[36] Hedayat S, Arefi M. [The effect of cognitive-behavioral therapy on improving sleep quality in the elderly (Persian)]. Knowledge \& Research in Applied Psychology. 2015; 16(2):60-8. https:// www.sid.ir/en/journal/ViewPaper.aspx?id=454469

[37] Edinger JD, Sampson WS. A primary care "friendly" cognitive behavioral insomnia therapy. Sleep. 2003; 26(2):177-82. [DOI:10.1093/sleep/26.2.177] [PMID]

[38] Morin CM, Hauri PJ, Espie CA, Spielman AJ, Buysse DJ, Bootzin RR. Nonpharmacologic treatment of chronic insomnia. Sleep. 1999; 22(8):1134-56. [DOI:10.1093/sleep/22.8.1134] [PMID]

[39] Torabi A, Ilali ES, Emadian SO, Mousavinasab N. Comparison of the effect of sleep hygiene education with the cognitive and behavioral method on the quality of sleep in elderly. Journal of Gerontology. 2019; 4(2):9-17. [DOI:10.29252/joge.4.2.9] 